\title{
Effect of minoxidil concentration on the deposition of drug and vehicle into the skin
}

\author{
Jui-Chen Tsai ${ }^{\text {a }}$, Gordon L. Flynn ${ }^{a}$, Norman Weiner a and James J. Ferry ${ }^{b}$ \\ ${ }^{a}$ College of Pharmacy, University of Michigan, Ann Arbor, MI 48109-1065 (USA) and 'b The Upjohn Company, Kalamazoo, \\ MI 49001 (USA)
}

(Received 28 March 1992)

(Modified version received 29 December 1992)

(Accepted 6 January 1993)

Key words: Minoxidil; Concentration effect; Drug deposition; Vehicle deposition; Skin

\begin{abstract}
Summary
Minoxidil dissolved in propylene glycol/ethanol/water $(20: 60: 20 ; \mathrm{v} / \mathrm{v})$ was more efficiently delivered into hairless mouse skin from a $0.02 \%$ solution than from a $2 \%$ formulation during in vitro mass balance experiments. Evaporation of the vehicle after application of the $2 \%$ minoxidil solution led to compositional changes so drastic that saturation is attained quickly. The shifting compositions of the vehicle were experimentally profiled and the drug's solubility was measured in media reflecting these changes over time. The original $2 \%$ formulation has excess capacity to dissolve minoxidil but, following its application, the solution rapidly saturates through evaporation, reaching saturation within 30 minutes. While the $2 \%$ formulation definitely functions at a proportionally higher thermodynamic activity than the $0.02 \%$ formulation at early times, most of this thermodynamic advantage is lost abruptly upon precipitation of the drug. The driving force following drug precipitation only reflects the dissolved minoxidil, and thus the relative delivery rate of the $2 \%$ formulation eventually drops to only several multiples of the delivery rate of the $0.02 \%$ solution which never quite attains saturation.
\end{abstract}

\section{Introduction}

The driving force for passive transport through a membrane is the chemical potential gradient expressed across the membrane. To create the gradient necessary to deliver a drug across the skin, one normally dissolves a drug in a solvent or vehicle to establish a certain concentration, and thus activity, of the drug at the outer surface of

Correspondence to: $\mathrm{N}$. Weiner, College of Pharmacy, University of Michigan, Ann Arbor, MI 48109-1065, U.S.A. the skin. Since different vehicles have different capacities to dissolve the drug, at any fixed level of activity one can have different concentrations of the drug at the interface of application depending on the solvency of the vehicle. Conversely, at a given concentration, the driving force, and hence the flux, depends on the vehicle's ability to dissolve the drug.

In most topical delivery situations one wishes to maximize flux from the formulation, for the usual problem is that one has difficulty getting sufficient drug into the skin to produce a desired effect. The simplest and most reliable way to 
maximize delivery is to work with saturated vehicles for, in principle, these operate at unit activity (solid state activity), which is the highest practically achievable thermodynamic activity. However, one may be able to further increase the delivery of drug into the skin by facilitating supersaturation as long as this state develops following topical application of the product. Judicious blending of volatile components can be used to achieve this end. As demonstrated by Coldman et al. (1969), the penetration of fluocinolide and fluocinolone acetonide from vehicles containing different proportions of isopropanol and either propylene glycol or isopropyl myristate was enhanced to a maximum as the volatile cosolvent isopropanol was allowed to evaporate. The increase in skin penetration is accounted for by an increase in the thermodynamic activity of the steroid in the concentrate formed from the vehicle. In studies by Tanaka et al. (1985), the deposition of hydrocortisone 17-butyrate 21-propionate from an oil-in-water cream and from several aqueous gels containing propylene glycol into silicone rubber was less under open conditions than under closed conditions. However, drug release across the polydimethysiloxane membrane from hydroalcoholic gels under open conditions was greater than under closed conditions. These differences were ascribed to evaporation-driven changes in the thermodynamic activity of the drug in the vehicle.

We too have studied mixed solvents as vehicles and, in our previous mass balance study with $2 \%$ minoxidil in propylene glycol/ethanol/water $(20: 60: 20, \mathrm{v} / \mathrm{v}$; Tsai et al., 1992), we reported that not only did the ethanol and water evaporate, but so did the ostensibly non-volatile propylene glycol. Though propylene glycol's evaporation rate was relatively independent of the amount of formula applied, the increase in minoxidil's concentration accompanying the evaporation of propylene glycol was particularly rapid when the formulation was applied as a thin film on the skin. Since the commercial minoxidil formulation barely contains enough propylene glycol to keep all the minoxidil in solution as the ethanol and water evaporate, and since propylene glycol itself is absorbed and evaporates, precipitation of the drug is inevitable. To demonstrate the effect of cvaporation on thcrmodynamic activity both in absence and in the presence of the confounding effects of drug precipitation, we have compared the deposition of drug and vehicle into the skin from solutions where precipitation of drug does and does not occur at various times after topical application of a thin film.

\section{Materials and Methods}

In vitro mass balance study with $0.02 \%$ minoxidil formulations

The test formulation consisted of $0.02 \% \mathrm{w} / \mathrm{v}$ minoxidil in a vehicle of $20 \%$ propylene glycol, $60 \%$ ethanol and $20 \%$ water by volume. This vehicle was spiked with $\left[{ }^{14} \mathrm{C}\right]$ propylene glycol and $\left[{ }^{3} \mathrm{H}\right]$ minoxidil. The resulting formulation contained approx. $0.5 \mu \mathrm{Ci}\left[{ }^{3} \mathrm{H}\right]$ minoxidil and $0.4 \mu \mathrm{Ci}$ $\left[{ }^{14} \mathrm{C}\right]$ propylene glycol per $\mathrm{ml}$. Mass balance of each of these species was assessed using procedures as described earlier (Tsai et al., 1992). Hairless mouse skin was used in the study. A volume of $62.5 \mu \mathrm{l}$ of the $0.02 \%$ minoxidil formulation was applied to skin sections having an exposed area of $1.77 \mathrm{~cm}^{2}\left(\sim 35 \mu \mathrm{l} / \mathrm{cm}^{2}\right)$. At various times, the system's components (residual formulation, skin strata and receiver) were analyzed for ${ }^{14} \mathrm{C}$ and ${ }^{3} \mathrm{H}$ by the methods previously described by Tsai et al. (1992).

\section{Determination of vehicle composition changes due to evaporation}

Two separate $2 \% \mathrm{w} / \mathrm{v}$ solutions of minoxidil in propylene glycol/ethanol/water $(20: 60: 20, \mathrm{v} / \mathrm{v})$ were prepared, one spiked with $\left[{ }^{3} \mathrm{H}\right]$ water and $\left[{ }^{14} \mathrm{C}\right]$ propylene glycol and the other spiked with $\left[{ }^{3} \mathrm{H}\right]$ water and $\left[{ }^{14} \mathrm{C}\right]$ ethanol. Evaporation of individual components of the vehicle was allowed to proceed from applications to a tissue culture multi-well plate (Flow Laboratories, McLean, VA) having flat-bottom, $2 \mathrm{~cm}^{2}$ area wells. The plate is a non-absorbing material and thus all loss of the solvents is through evaporation. The temperature was maintained at $25^{\circ} \mathrm{C}$ by use of a water bath (Precision Scientific, Chicago, IL). In these studies, $40 \mu \mathrm{l}$ of the formulations were 
added to the wells $\left(20 \mu \mathrm{l} / \mathrm{cm}^{2}\right)$. At designated intervals, substance remaining in each well was washed into a counting vial with $1 \mathrm{ml}$ of $70 \%$ alcohol. The washing was repeated three more times and scintillation cocktail (Ecolite ${ }^{\mathrm{TM}}+$ ); ICN Biomedicals, Irvine, CA) was added and the samples were assayed in a liquid scintillation counter (Beckman LS9000, Beckman Instrument, Fullerton, CA).

\section{Minoxidil solubility measurement}

Minoxidil's solubility was measured in solvent compositions representing those formed in the evaporation experiments. Excess minoxidil was equilibrated with each solvent mixture for at least $72 \mathrm{~h}$ at $25^{\circ} \mathrm{C}$. To facilitate equilibrium, the samples $(n=3-5)$ were continuously shaken in a water bath (Precision Scientific, Chicago, IL). Aliquots were removed and centrifuged to separate and remove all particulate drug. After appropriate dilution with water, these were assayed spectrophotometrically at $228.7 \mathrm{~nm}$ (Lambda 7 Spectrophotometer, Perkin-Elmer, Oak Brook, IL).

\section{Results}

The results of the in vitro mass balance experiments for the 0.02 and $2 \%$ minoxidil formulations are shown in Figs 1-5. In terms of percentages, both minoxidil and propylene glycol are lost from the donor compartment at a faster rate from the $0.02 \%$ formulation than from the $2 \%$ formulation (Fig. 1). There is almost no propylene glycol left in the donor compartment at $20 \mathrm{~h}$, a time at which the disappearance of minoxidil has all but ceased from the donor compartment. It thus appears that one needs solvent in the donor compartment to maintain a solution state for the drug and allow its dissolution and subsequent partitioning into the skin. The percentages of minoxidil and propylene glycol found in the epidermis (Fig. 2) are approximately the same for the 2 and $0.02 \%$ formulations up to $2 \mathrm{~h}$. At longer intervals, a higher percentage of minoxidil accumulates in the epidermis from the $0.02 \%$ formulation. The epidermal drug concentration peaks near $35 \%$
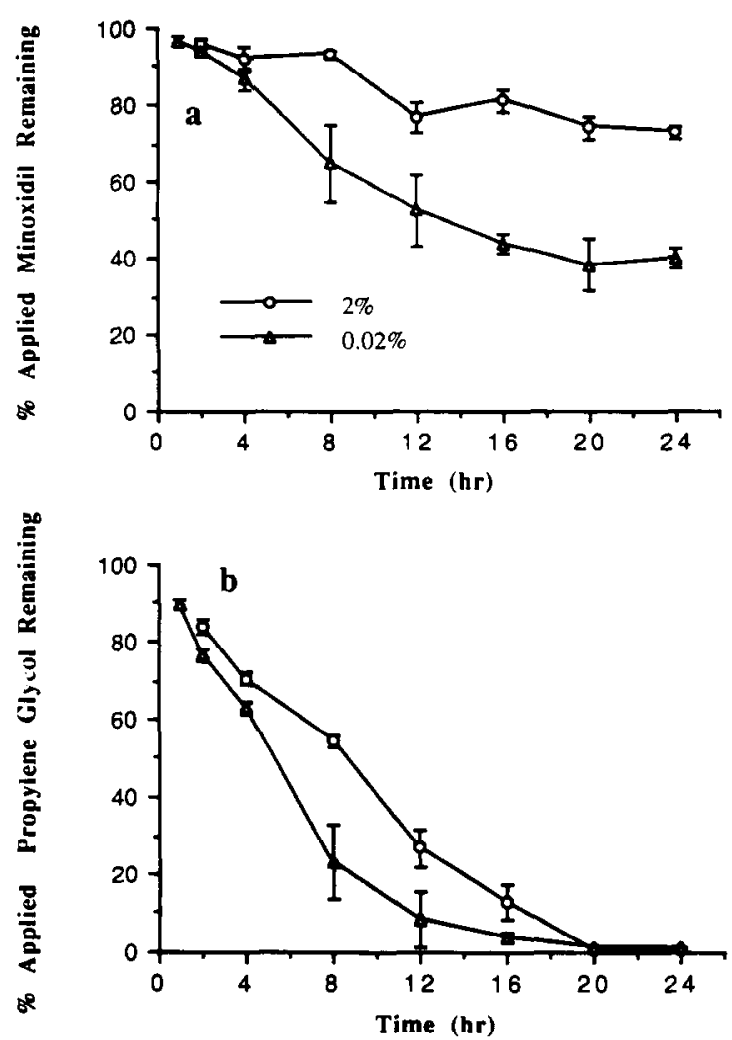

Fig. 1. The percentage of minoxidil and propylene glycol remaining in the donor side of the diffusion cell as a function of time when $62.5 \mu \mathrm{l}$ of 2 and $0.02 \%$ minoxidil solution in propylene glycol/ethanol/water $(20: 60: 20 \mathrm{v} / \mathrm{v})$ were applied. (a) Minoxidil; (b) propylene glycol. Each data point represents mean $\pm \mathrm{SE}(n=3-5)$.

around the $16 \mathrm{~h}$ time point. In contrast, the drug level in the epidermis from the $2 \%$ formulation plateaued by the 12th hour, and only about $15 \%$ of the drug was deposited. For propylene glycol, the peak level in the skin seemed to be reached at earlier times with the $0.02 \%$ formulation. Unlike the situation with minoxidil, propylene glycol was systematically depleted from the epidermis until virtually none remained at the $20 \mathrm{~h}$ time point.

The percentages of minoxidil and propylene glycol found in the dermis with time are shown in Fig. 3. Consistent with the epidermal results, between the 4 and $20 \mathrm{~h}$ time points, a greater percentage of minoxidil was found in the dermis for the $0.02 \%$ formulation. Propylene glycol con- 
centrations peaked earlier but otherwise behaved similarly to minoxidil and notable differences in the dermal percentages, favoring the $0.02 \%$ formulation, appeared between the 2 and 12 h time points. Interestingly, for both minoxidil and propylene glycol, the dermal percentages of the two strengths of minoxidil came into coincidence towards the end of the $24 \mathrm{~h}$ application period.

Amounts of minoxidil and propylene glycol permeating into the receiver compartment are shown in Fig. 4. Little drug permeates in the first $4 \mathrm{~h}$. At later times, receiver accumulation of propylene glycol and minoxidil is greater for the $0.02 \%$ formulation. More than $25 \%$ of minoxidil penetrated into the receiver from the $0.02 \%$ solution over the $24 \mathrm{~h}$ course of the experiments, a value fully 5 -times that observed for the $2 \%$ solu-
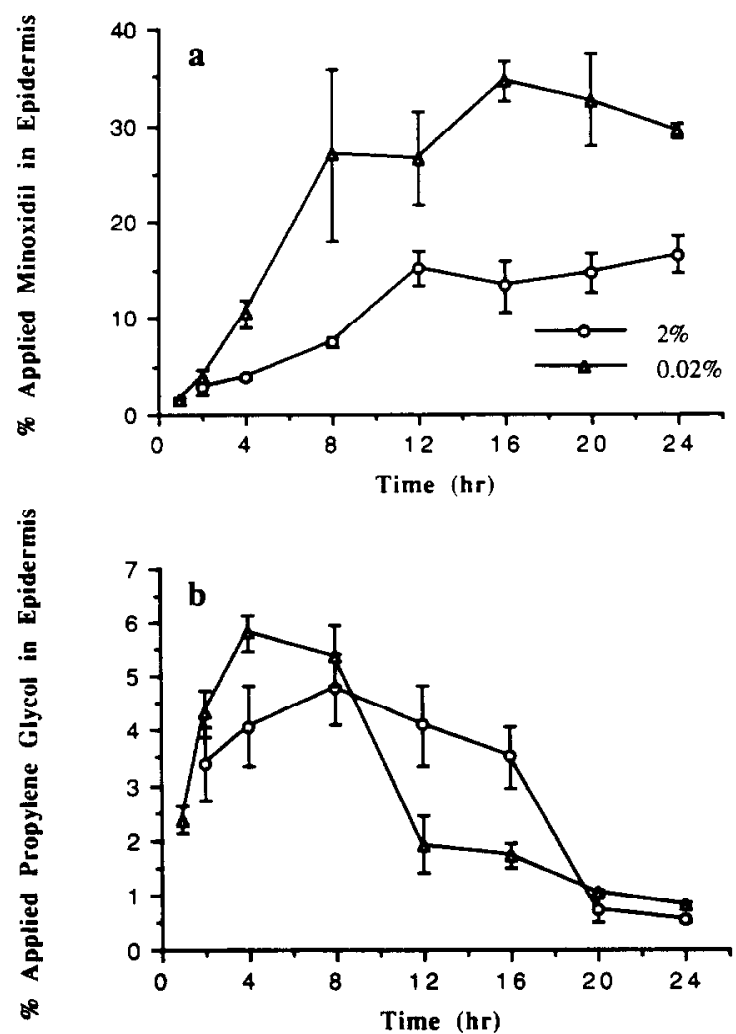

Fig. 2. The percentage of minoxidil and propylene glycol found in the epidermis as a function of time when $62.5 \mu 1$ of 2 and $0.02 \%$ minoxidil solution in propylene glycol/ethanol/ water $(20: 60: 20 \mathrm{v} / \mathrm{v})$ were applied. (a) Minoxidil; (b) propylene glycol. Each data point represents mean \pm SE $(n=3-5)$.
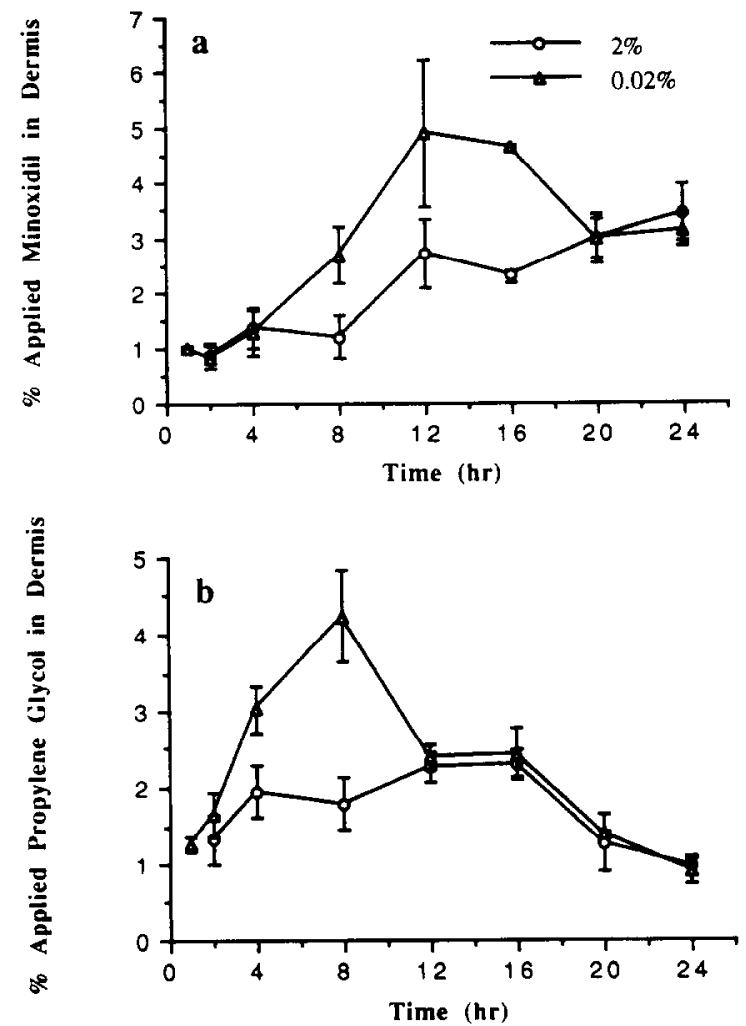

Fig. 3. The percentage of minoxidil and propylene glycol found in the dermis as a function of time when $62.5 \mu \mathrm{l}$ of 2 and $0.02 \%$ minoxidil solution in propylene glycol/ethanol/ water $(20: 60: 20 \mathrm{v} / \mathrm{v})$ were applied. (a) Minoxidil; (b) propylene glycol. Each data point represents mean $\pm \operatorname{SE}(n=3-5)$.

tion. For propylene glycol, total $24 \mathrm{~h}$ accumulations from the two minoxidil formulations were similar $(\sim 50 \%)$ but with the limiting level being reached much faster from the $0.02 \%$ solution. There is even a hint that the receiver propylene glycol level passes through a maximum in the course of the experiments, which makes sense only if propylene glycol is back diffusing and evaporating from the surface of the skin.

Upon combining the information found in Figs $1-4$, one finds complete mass balance $(98-100 \%)$ for minoxidil at both formulation strengths but systematic loss of propylene glycol which reached a total of about $50 \%$ by $24 \mathrm{~h}$. All the evidence points to the fact that this solvent is evaporating, albeit more slowly than ethanol and water. The net vehicle compositions expressed in volume 
TABLE 1

Percent saturation of minoxidil in the remaining solution due to solvent evaporation from $2 \% \mathrm{w} / v$ minuxidil in propylene glycol / ethanol / water (20:60:20 v/v) with $40 \mu \mathrm{l} / 2 \mathrm{~cm}^{2}$ applied on tissue culture plate at $25^{\circ} \mathrm{C}$

\begin{tabular}{|c|c|c|c|c|c|c|}
\hline \multirow[t]{2}{*}{ Time } & \multirow{2}{*}{$\begin{array}{l}\text { Total } \\
\text { remaining }(\mu \mathrm{l})\end{array}$} & \multicolumn{3}{|c|}{ Volume fraction } & \multirow{2}{*}{$\frac{\text { Solubility }(\mathrm{mg} / \mathrm{ml})}{(\text { mean } \pm \mathrm{SD})}$} & \multirow[t]{2}{*}{$\%$ saturation } \\
\hline & & Water & Ethanol & Propylene glycol & & \\
\hline $0 \mathrm{~min}$ & 40.0 & 0.20 & 0.60 & 0.20 & $56.3 \pm 0.7$ & 35.5 \\
\hline $10 \mathrm{~min}$ & 25.9 & 0.19 & 0.50 & 0.31 & $62.4 \pm 1.3$ & 49.5 \\
\hline $20 \mathrm{~min}$ & 14.6 & 0.19 & 0.25 & 0.56 & $66.8 \pm 0.5$ & 81.9 \\
\hline $30 \mathrm{~min}$ & 11.0 & 0.17 & 0.09 & 0.74 & $69.1 \pm 0.8$ & 105.5 \\
\hline $40 \mathrm{~min}$ & 9.9 & 0.13 & 0.05 & 0.82 & $75.1 \pm 1.3$ & 108.2 \\
\hline $1 \mathrm{~h}$ & 9.0 & 0.06 & 0.05 & 0.89 & $79.3 \pm 1.0$ & 112.3 \\
\hline $1.5 \mathrm{~h}$ & 8.6 & 0.03 & 0.05 & 0.92 & $86.7 \pm 1.0$ & 106.9 \\
\hline $2 \mathrm{~h}$ & 8.4 & 0.01 & 0.05 & 0.94 & $85.8 \pm 2.3$ & 110.7 \\
\hline $3 \mathrm{~h}$ & 8.2 & 0.00 & 0.05 & 0.95 & & \\
\hline $4 \mathrm{~h}$ & 8.0 & 0.00 & 0.05 & 0.95 & & \\
\hline $6 \mathrm{~h}$ & 7.8 & 0.00 & 0.05 & 0.95 & & \\
\hline $8 \mathrm{~h}$ & 7.5 & 0.00 & 0.05 & 0.95 & $88.0 \pm 0.6$ & 121.4 \\
\hline
\end{tabular}

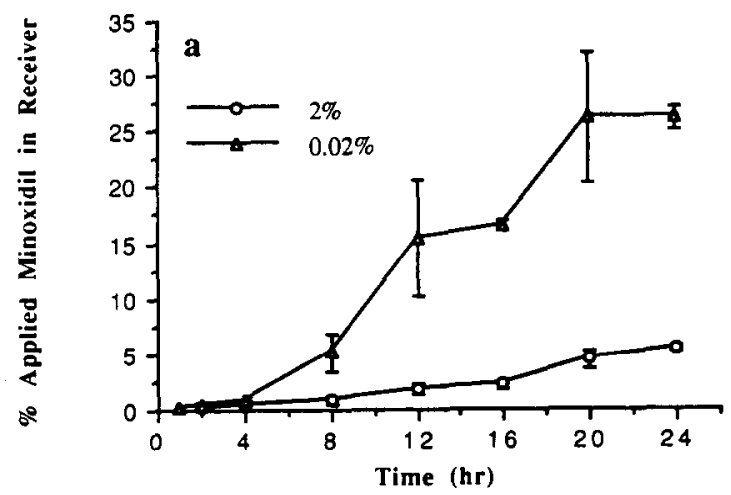

fractions as a function of time for $2 \%$ minoxidil solution maintained at $25^{\circ} \mathrm{C}$ in the wells of the tissue culture plate are shown in Table 1. A ternary phase diagram describing the vehicle composition changes as a function of time clearly shows the solvent composition moves rapidly towards neat propylene glycol (Fig. 5). Minoxidil is $35 \%$ saturated (a reflection of thermodynamic activity) in the original $2 \%$ formulation. It is rapidly saturated through evaporation, reaching the saturation level within $30 \mathrm{~min}$. Thereafter, it supersaturates and eventually precipitates. In the

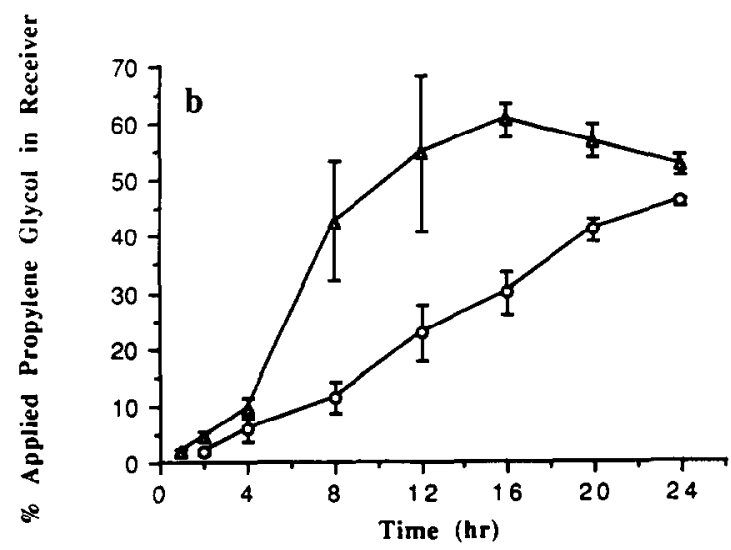

Fig. 4. The percentage of minoxidil and propylene glycol found in the receiver as a function of time when $62.5 \mu 1$ of 2 and $0.02 \%$ minoxidil solution in propylene glycol/ethanol/ water $(20: 60: 20 \mathrm{v} / \mathrm{v})$ were applied. (a) Minoxidil; (b) propylene glycol. Each data point represents mean \pm SE $(n=3-5)$.

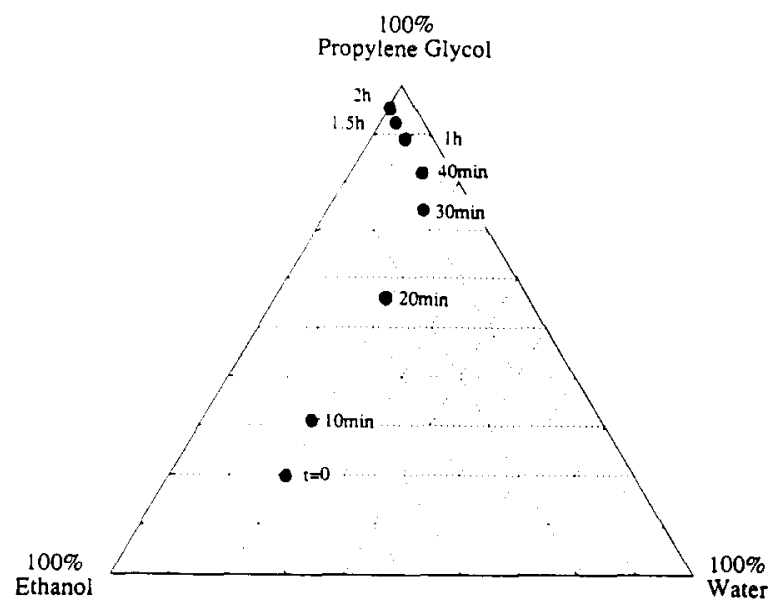

Fig. 5. Phase diagram of vehicle compositional changes as a function of time due to evaporation. 
evaporation experiments, no precipitation was observed at $8 \mathrm{~h}$.

\section{Discussion}

Wester and Maibach (1976) investigated the relationship between topical dose applied and percutaneous absorption for several drugs in the Rhesus monkey and in man. When the compounds were applied in either acetone (testosterone, hydrocortisone) or methanol (benzoic acid), the percentages absorbed were greater for the lesser concentrations. However, as the concentration of drug increased, the absolute amount of drug $(\mu \mathrm{g})$ absorbed also always increased. A similar result was reported by Scheuplein and Ross (1974). The present study represents a more detailed exploration of such effects.

The amount of vehicle remaining on the surface of the skin as a function of time is determined by the respective evaporation rates of its component solvents and their respective uptake into the skin. Since solutes affect the thermodynamic activities of the solvents in which they are dissolved, one expects the presence of solute to alter solvent evaporation and permeation rates. Such effects are particularly noted in the data appearing in Fig. 4b.

Even in the absence of permeation of the solvents, when placed on an inert surface, the $2 \%$ $\mathrm{w} / \mathrm{v}$ minoxidil solution undergoes vehicle composition changes through evaporation sufficient to lead to a saturated minoxidil solution within 30 $\min$ at $25^{\circ} \mathrm{C}$. On the skin's surface, with permeation and absorption of the vehicle occurring concurrently and with insensible perspiration contributing moisture and thereby diluting the film, the saturation process should be even faster (Fig. 6). The higher temperature of the skin surface (approx. $32^{\circ} \mathrm{C}$ ) further accelerates the evaporation and quite possibly the saturation process itself. Clearly, the time to achieve saturation is application volume dependent since the evaporation process is zero-order and it therefore takes longer for a thicker layer to reach the saturation point. Since the concentration of $0.02 \%$ minoxidil is 100 -times lower than the clinical formulation,

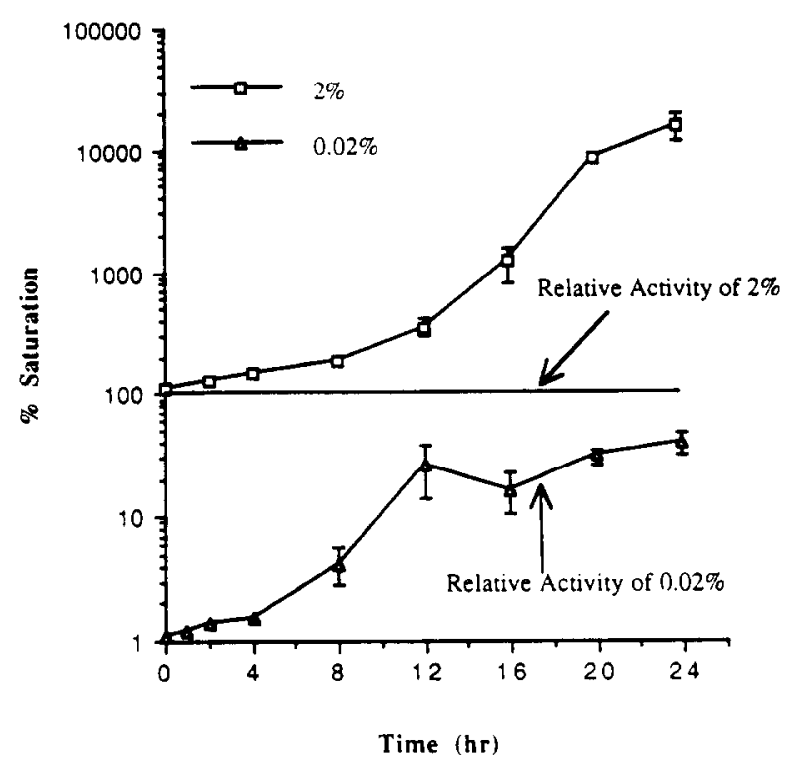

Fig. 6. The calculated percent saturation of minoxidil in the remaining formulation on the skin surface as a function of time for 2 and $0.02 \%$ formulation. Data were derived from Fig. 1 and calculated based on the solubility of minoxidil in propylene glycol of $90 \mathrm{mg} / \mathrm{ml}$ assuming ethanol and water evaporated instantaneously upon application.

the former solution never actually attains saturation in $24 \mathrm{~h}$. In support of these contentions, an analysis of the percent saturation of minoxidil in the 0.02 and $2 \%$ formulations following their placement on the skin surface is shown in Fig. 6. The calculations are based on the measured solubility of minoxidil in propylene glycol of 90 $\mathrm{mg} / \mathrm{ml}$. For convenience, it is assumed that ethanol and water cvaporate instantaneously upon making the application, an assumption which does no harm as these more volatile solvents are virtually exhausted by the time saturation is at hand. The onset of supersaturation on the skin surface is predicted for the $2 \%$ formulation. By the $24 \mathrm{~h}$ point, the calculated level of supersaturation is 100 -fold and a logarithmic scale is required to show the trend. There are no precedents for such exaggerated levels of supersaturation being achieved and sustained and, in the specific instance of minoxidil, the previous work of Chiang et al. (1985) demonstrates its precipitation promptly follows its supersaturation. Hence, it is quite reasonable to assume that minoxidil perme- 
ation is from a saturated suspension in the case of the initially $2 \%$ solution and that constant thermodynamic activity is maintained through the entire course of drug delivery. On the other hand, the percent saturation of minoxidil on the skin surface from the $0.02 \%$ solution gradually and continuously increases but never attains $50 \%$ of the saturation level during the experiment. Here it seems reasonable to assume that thermodynamic activity of the drug is constantly increasing without the drug's precipitation.

Considering the above, the increased efficiency of delivery of minoxidil from the low concentration formulation has the following straightforward explanation. While the $2 \%$ formulation must act at a proportionally higher thermodynamic activity than the $0.02 \%$ formulation for a period of time, its advantage disappears substantially and abruptly upon precipitation of the drug. The driving force following minoxidil's precipitation no longer reflects all the minoxidil present, and thus the delivery rate from the $2 \%$ formulation drops to only several multiples of that of the $0.02 \%$ solution. Consequently, even though the absolute amounts of minoxidil deposited into the various skin strata and receiver compartment are always greater for the $2 \%$ formulation than for the $0.02 \%$ formulation, eventually one has one-hundred times the total drug producing only a 2-fold higher flux (saturation vs half-saturation at the $24 \mathrm{~h}$ point). When delivery is looked at in terms of the full 24 hour experimental span, the 100-fold higher initial concentration only delivers 20 -fold more total drug.

\section{Acknowledgment}

This study was supported by the Upjohn Company, Kalamazoo, MI.

\section{References}

Chiang, C.M., Flynn, G.L., Weiner, N.D. and Szpunar, G.J., Bioavailability assessment of topical delivery systems: Effect of vehicle evaporation upon in vitro delivery of minoxidil from solution formulations. Int. J. Pharm., 55 (1989) 229-235.

Coldman, M.F., Poulsen, B.J. and Higuchi, T, Enhancement of percutaneous absorption by the use of volatile:nonvolatile systems as vehicles. J. Pharm. Sci., 58 (1969) 10981102.

Scheuplein, R.J. and Ross, L.W., Mechanism of percutaneous absorption: V. Percutaneous absorption of solvent deposited solids. J. Invest. Dermatol., 62 (1974) 353-360.

Tanaka, S., Takashima, Y., Murayama, H. and Tsuchiua, S., Studies on drug release from ointments: V. Release of hydrocortisone butyrate propionate from topical dosage forms to silicone rubber. Int. J. Pharm., 27 (1985) 20-38.

Tsai, J.-C., Cappel, M.J., Flynn, G.L., Weincr, N.D., Kreuter, J. and Ferry, J.J., Drug and vehicle deposition from topical applications: In vitro mass balance technique using minoxidil solutions. J. Pharm. Sci., 81 (1992) 736-743.

Wester, R.C. and Maibach, H.I., Relationship of topical dose and percutaneous absorption in rhesus monkey and man. J. Invest. Dermatol., 67 (1976) 518-520. 